\title{
Efficient method of two-dimensional DOA estimation for coherent signals
}

\author{
Heping Shi ${ }^{1}$, Zhuo Li2 ${ }^{2,5^{*}}$, Dun Liü ${ }^{3}$ and Hua Chen ${ }^{4}$
}

\begin{abstract}
An effective decoherence method called the fourth-order cumulants-based improved Toeplitz matrices reconstruction (FOC-ITMR) is addressed for two-dimensional (2-D) direction-of-arrival (DOA) estimation of coherent signals. To avoid the loss of the array's physical aperture, the FOC-ITMR method fully utilizes the information of received data from the whole two parallel uniform linear arrays (ULAs) and the changing reference element based on FOC. Compared with previous works, the proposed method can offer excellent decoherence performance in both white noise and color noise environments. In addition, the proposed algorithm can achieve automatic pair-matching without additional computation. The theoretical analysis and simulation results confirm the effectiveness of the proposed algorithm.
\end{abstract}

Keywords: Direction-of-arrival (DOA), Two-dimensional (2-D), Coherent sources, Fourth-order cumulants (FOC)

\section{Introduction}

Direction-of-arrival (DOA) estimation is a major research issue in array signal-processing applications such as radar, wireless communication, and sonar [1-5]. Among different DOA estimation methods, 2-D DOA estimation of coherent source signals [6-10] has drawn increasing attentions. Conventional high-precision methods, such as MUSIC [11] and ESPRIT [12], have achieved exciting performance. Unfortunately, these algorithms are easy to affect by noise as well as require its prior knowledge. Besides, the total number of incident signals must be less than that of the sensors [13]. Fortunately, the high-order cumulants, such as the fourth-order cumulants (FOC), have been shown to be a promising method since the noise covariance, which is Gaussian distributed, can be ignored [14-16]. Furthermore, another key motivation of using the FOC is the ability to resolve more number of sources than or equal to that of array elements [17, 18].

In practical, highly correlated or coherent signals are everywhere in multipath propagation environments due to reflection and refraction of source signals. Based on such scenario, the coherent sources facilitate the rank loss of the covariance matrix, which could result in the failure of the conventional high-resolution algorithms. To achieve signal decorrelation,

\footnotetext{
*Correspondence: zli@tju.edu.cn

${ }^{2}$ Tianjin Key Laboratory of Wireless Mobile Communications and Power

Transmission, Tianjin Normal University, Tianjin 300387, China

${ }^{5} \mathrm{College}$ of Electronic and Communication Engineering, Tianjin Normal

University, Tianjin 300387, China

Full list of author information is available at the end of the article
}

the spatial smoothing (SS) [19] and forward-backward spatial smoothing (FBSS) [20] are especially noteworthy, which achieve coherent sources location. Chen et al. [21] have proposed a 2-D ESPRIT-like algorithm to attain decorrelation. Based on three correlation matrices, Wang et al. [22] have presented a 2-D DOA estimation method. Recently, Nie et al. [23] have introduced a computationally efficient subspace method with L-shaped array. In [24], an effective 2-D DOA estimation method using a sparse L-shaped array is proposed to obtain good estimation performance and less computational complexity. In [25], the FOC-FSS approach has been presented to remedy rank deficiency problem. In [26], the FOC-TMR method is presented to obtain source location by reconstructing two Toeplitz matrices.

In this paper, a new Toeplitz matrices reconstruction algorithm, called the FOC-ITMR, is proposed for 2-D coherent signals DOA estimation. The information of the whole two subarrays elements is fully utilized to reconstruct two new matrices by changing the reference element, which can achieve the decorrelation of the coherent signals as well as avoid the loss of array aperture.

\section{Signal model}

As illustrated in Fig. 1, the antenna array consists of two parallel ULAs $\left(X_{a}\right.$ and $\left.Y_{a}\right)$ in the $x-y$ plane. Each ULA has $N$ identical omni-directional sensors with spacing $d_{x}$, and the interelement spacing between the two ULAs is $d_{y}$.

Suppose that $P$ far-field narrowband source signals $s_{i}(t)(i=1, \ldots, P)$ impinge on the two parallel ULAs from 


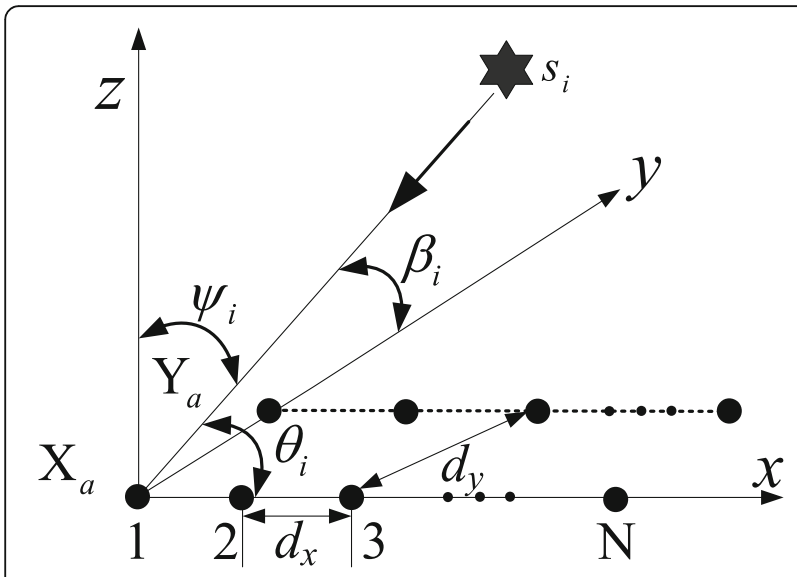

Fig. 1 Parallel array configuration for 2-D DOA estimation

directions $\left(\theta_{i}, \beta_{i}\right)$, where $\theta_{i}$ and $\beta_{i}$ are measured relatively to the $x$ and $y$ axes corresponding to the $i$ th signal, respectively. Thus, $\psi_{i}$, the DOA of the $i$ th signal relative to the $z$ axis, can be written by

$$
\cos ^{2} \theta_{i}+\cos ^{2} \beta_{i}+\cos ^{2} \psi_{i}=1
$$

Here, $\mathbf{X}_{i}=\left(\theta_{i}, \beta_{i}, \psi_{i}\right)$ are termed as 2-D DOA vector angles. Let the $m$ th element of the subarray $X_{a}$ be the phase reference, and then the observed signals $x_{k}^{m}(t)$ at the $k$ th element in time $t$ can be expressed as

$$
\begin{aligned}
x_{k}^{m}(t)= & \sum_{i=1}^{Q} \gamma_{i} s_{1}(t) e^{-j \frac{2 \pi}{\lambda} d_{x}(k-m) \cos \theta_{i}} \\
& +\sum_{i=Q+1}^{P} s_{i}(t) e^{-j \frac{2 \pi}{\lambda} d_{x}(k-m) \cos \theta_{i}}+n_{x, k}(t)
\end{aligned}
$$

where the superscript $m(m=1,2, \cdots, N)$ of the $x_{k}^{m}(t)$ stands for the number of the reference element in subarray $X_{a}$, and the subscript $k(k=1,2, \cdots, N)$ of the $x_{k}^{m}(t)$ denotes the number of the element along the $x$ positive axis in subarray $X_{a} \cdot n_{x, k}(t)$ is the additive Gaussian noise of the $k$ th element in subarray $X_{a}$ and $\gamma_{i}$ indicates the amplitude fading factor of the first $Q$ correlative signals (without loss of generality, we assume $\gamma_{1}=1$ ).

With a similar processing, employing the $m$ th element of the subarray $Y_{a}$ as the phase reference, and then the observed signals $y_{k}^{m}(t)$ at the $k$ th element in time $t$ can be expressed as

$$
\begin{aligned}
y_{k}^{m}(t)= & \sum_{i=1}^{Q} \gamma_{i} s_{1}(t) e^{-j \frac{2 \pi}{\lambda} d_{x}(k-m) \cos \theta_{i}} e^{j \frac{2 \pi}{\lambda} d_{y} \cos \beta_{i}} \\
& +\sum_{i=Q+1}^{P} s_{i}(t) e^{-j \frac{2 \pi}{\lambda}} d_{x}(k-m) \cos \theta_{i} e^{j \frac{2 \pi}{\lambda} d_{y} \cos \beta_{i}}+n_{y, k}(t)
\end{aligned}
$$

The observed vectors $\mathbf{X}^{m}(t)$ and $\mathbf{Y}^{m}(t)$ can be written as

$$
\begin{aligned}
& \mathbf{X}^{m}(t)=\left[x_{1}^{m}(t), x_{2}^{m}(t), \cdots, x_{N}^{m}(t)\right]^{T} \\
& \mathbf{Y}^{m}(t)=\left[y_{1}^{m}(t), y_{2}^{m}(t), \cdots, y_{N}^{m}(t)\right]^{T}
\end{aligned}
$$

\section{Angle estimation algorithm}

\subsection{The FOC-ITMR algorithm}

The new proposed algorithm named as FOC-ITMR, which is based on reconstructing two Toeplitz matrices $\mathbf{C}_{1}$ and $\mathbf{C}_{2}$ by using two parallel ULAs, is described in detail in this subsection. Firstly, we define $\mathbf{C}_{1}$ and $\mathbf{C}_{2}$ with the cumulant elements $c_{k}^{m}$ and $\tilde{c}_{k}^{m}$ arranging as follows

$$
\begin{aligned}
& c_{k}^{m}=\operatorname{cum}\left[x_{m}^{m}(t),\left(x_{m}^{m}(t)\right)^{*},\left(x_{m}^{m}(t)\right)^{*}, x_{k}^{m}(t)\right] \\
& =\operatorname{cum}\left[\sum_{i=1}^{Q} \gamma_{i} s_{1}(t)+\sum_{i=Q+1}^{P} s_{i}(t)+n_{x, 0}(t), \sum_{i=1}^{Q} \gamma_{i}^{*} s_{1}^{*}(t)\right. \\
& +\sum_{i=Q+1}^{P} s_{i}^{*}(t)+n_{x, 0}(t), \sum_{i=1}^{Q} \gamma_{i}^{*} s_{1}^{*}(t)+\sum_{i=Q+1}^{P} s_{i}^{*}(t) \\
& +n_{x, 0}{ }^{*}(t), \sum_{i=1}^{Q} \gamma_{i} s_{1}(t) e^{-j \frac{2 \pi}{\lambda} d_{x}(k-m) \cos \theta_{i}} \\
& \left.+\sum_{i=Q+1}^{P} s_{i}(t) e^{-j \frac{2 \pi}{\lambda} d_{x}(k-m) \cos \theta_{i}}+n_{x, k}(t)\right] \\
& =\left(\sum_{i=1}^{Q} \gamma_{i}^{*}\right)^{2}\left(\sum_{i=1}^{Q} \gamma_{i}\right) \sum_{i=1}^{Q} \gamma_{i} e^{-j \frac{2 \pi}{\lambda} d_{x}[(k-1)-(m-1)] \cos \theta_{i}} \\
& \operatorname{cum}\left[s_{1}(t), s_{1}^{*}(t), s_{1}^{*}(t), s_{1}(t)\right]+\sum_{i=Q+1}^{P} e^{-j \frac{2 \pi}{\lambda}} d_{x}[(k-1)-(m-1)] \cos \theta_{i} \\
& \operatorname{cum}\left[s_{i}(t), s_{i}^{*}(t), s_{i}^{*}(t), s_{i}(t)\right]=\sum_{i=1}^{P} d_{i} e^{-j \frac{2 \pi}{\lambda}} d_{x}[(k-1)-(m-1)] \cos \theta_{i} \\
& \tilde{c}_{k}^{m}=\operatorname{cum}\left[x_{m}^{m}(t),\left(x_{m}^{m}(t)\right)^{*},\left(x_{m}^{m}(t)\right)^{*}, y_{k}^{m}(t)\right] \\
& =\operatorname{cum}\left[\sum_{i=1}^{Q} \gamma_{i} s_{1}(t)+\sum_{i=Q+1}^{P} s_{i}(t)+n_{x, 0}(t), \sum_{i=1}^{Q} \gamma_{i}^{*} s_{1}^{*}(t)+\sum_{i=Q+1}^{P} s_{i}^{*}(t)+n_{x, 0}^{*}(t),\right. \\
& \sum_{i=1}^{Q} \gamma_{i}^{*} s_{1}^{*}(t)+\sum_{i=Q+1}^{P} s_{i}^{*}(t)+n_{x, 0}^{*}(t), \sum_{i=1}^{Q} \gamma_{i} s_{1}(t) e^{-j \frac{2 \pi}{\lambda} d_{x}(k-m) \cos \theta_{i}} e^{j \frac{2 \pi}{\lambda} d_{y} \cos \beta_{i}} \\
& \left.+\sum_{i=Q+1}^{P} s_{i}(t) e^{-j \frac{2 \pi}{\lambda} d_{x}(k-m) \cos \theta_{i}} e^{\frac{2 \pi}{\lambda} d_{y} \cos \beta_{i}}+n_{y, 0}(t)\right] \\
& =\left(\sum_{i=1}^{Q} \gamma_{i}^{*}\right)^{2}\left(\sum_{i=1}^{Q} \gamma_{i}\right) \sum_{i=1}^{Q} \gamma_{i} e^{-j \frac{2 \pi}{\lambda} d_{x}[(k-1)-(m-1)] \cos \theta_{i}} e^{\frac{2 \pi}{\lambda}} d_{y} \cos \beta_{i} \\
& \operatorname{cum}\left[s_{1}(t), s_{1}^{*}(t), s_{1}^{*}(t), s_{1}(t)\right] \\
& +\sum_{i=Q+1}^{p} e^{-\frac{2 \pi}{\lambda} d_{x}[(k-1)-(m-1)] \cos \theta_{i}} e^{\frac{2 \pi}{\lambda} d_{y} \cos \beta_{i}} \operatorname{cum}\left[s_{i}(t), s_{i}^{*}(t), s_{i}^{*}(t), s_{i}(t)\right] \\
& =\sum_{i=1}^{P} d_{i} e^{-j \frac{2 \pi}{\lambda}} d_{x}[(k-1)-(m-1)] \cos \theta_{i} e^{j \frac{2 \pi}{\lambda}} d_{y} \cos \beta_{i}
\end{aligned}
$$

where

$$
d_{i}=\left\{\begin{array}{cc}
\gamma_{i} \tilde{\rho}_{4, s_{1}} & i=1, \cdots, Q \\
\rho_{4, s_{i}} & i=Q+1, \cdots, P
\end{array}\right.
$$

with $\rho_{4, S_{i}}=\operatorname{cum}\left[S_{i}(t), S_{i}^{*}(t), S_{i}^{*}(t), S_{i}(t)\right]$ and $\tilde{\rho}_{4, S_{1}}=\left(\sum_{i=1}^{Q} \gamma_{i}^{*}\right)^{2}\left(\sum_{i=1}^{Q} \gamma_{i}\right) \rho_{4, S_{1}}$.

Then, the Toeplitz matrices $\mathrm{C}_{1}$ and $\mathrm{C}_{2}$ can be constructed, respectively, 


$$
\begin{aligned}
& \mathbf{C}_{1}=\left[\begin{array}{cccc}
c_{1}^{1} & c_{1}^{2} & \ldots & c_{1}^{N} \\
c_{2}^{1} & c_{2}^{2} & \ldots & c_{2}^{N} \\
\vdots & \vdots & \cdots & \vdots \\
c_{N}^{1} & c_{N}^{2} & \cdots & c_{N}^{N}
\end{array}\right] \\
& =\left[\begin{array}{cccc}
\sum_{i=1}^{P} d_{i} e^{-j \frac{2 \pi}{\lambda} d_{x}(0) \cos \theta_{i}} & \sum_{i=1}^{P} d_{i} e^{-j \frac{2 \pi}{\lambda} d_{x}(-1) \cos \theta_{i}} & \ldots & \sum_{i=1}^{P} d_{i} e^{-j \frac{2 \pi}{\lambda}} d_{x}(1-N) \cos \theta_{i} \\
\sum_{i=1}^{P} d_{i} e^{-j \frac{2 \pi}{\lambda} d_{x}(1) \cos \theta_{i}} & \sum_{i=1}^{P} d_{i} e^{-j \frac{2 \pi}{\lambda} d_{x}(0) \cos \theta_{i}} & \ldots & \sum_{i=1}^{P} d_{i} e^{-j \frac{2 \pi}{\lambda}} d_{x}(2-N) \cos \theta_{i} \\
\vdots & \ldots & \vdots \\
\sum_{i=1}^{P} d_{i} e^{-j \frac{2 \pi}{\lambda}} d_{x}(N-1) \cos \theta_{i} & \sum_{i=1}^{P} d_{i} e^{-j \frac{2 \pi}{\lambda} d_{x}(N-2) \cos \theta_{i}} & \ldots & \sum_{i=1}^{P} d_{i} e^{-j \frac{2 \pi}{\lambda}} d_{x}(0) \cos \theta_{i}
\end{array}\right] \\
& =\left[\begin{array}{cccc}
1 & 1 & \cdots & 1 \\
e^{-j \frac{2 \pi}{\lambda} d_{x}(1) \cos \theta_{1}} & e^{-j \frac{2 \pi}{\lambda} d_{x}(1) \cos \theta_{2}} & \cdots & e^{-j \frac{2 \pi}{\lambda} d_{x}(1) \cos \theta_{P}} \\
\vdots & \vdots & \cdots & \vdots \\
e^{-j \frac{2 \pi}{\lambda} d_{x}(N-1) \cos \theta_{1}} & e^{-j \frac{2 \pi}{\lambda} d_{x}(N-1) \cos \theta_{2}} & \cdots & e^{-j \frac{2 \pi}{\lambda} d_{x}(N-1) \cos \theta_{P}}
\end{array}\right]
\end{aligned}
$$

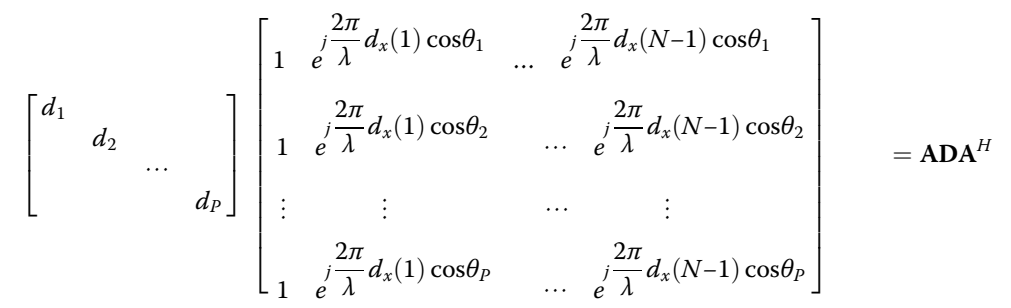$$
\mathbf{C}_{2}=\left[\begin{array}{cccc}
\tilde{c}_{1}^{1} & \tilde{c}_{1}^{2} & \ldots & \tilde{c}_{1}^{N} \\
\tilde{c}_{2}^{1} & \tilde{c}_{2}^{2} & \ldots & \tilde{c}_{2}^{N} \\
\vdots & \vdots & \cdots & \vdots \\
\tilde{c}_{N}^{1} & \tilde{c}_{N}^{2} & \cdots & \tilde{c}_{N}^{N}
\end{array}\right]
$$

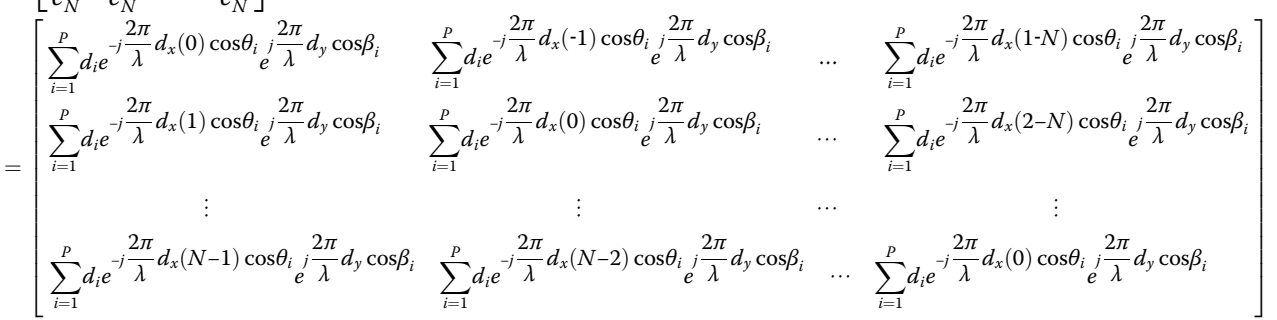

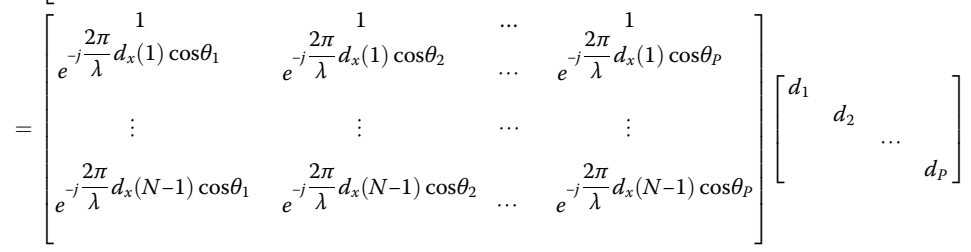$$
\left[\begin{array}{ccc}
e^{j \frac{2 \pi}{\lambda} d_{y} \cos \beta_{1}} & \\
e^{j \frac{2 \pi}{\lambda} d_{y} \cos \beta_{2}} & \\
\cdots & e^{j \frac{2 \pi}{\lambda} d_{y} \cos \beta_{P}}
\end{array}\right]\left[\begin{array}{cccc}
1 e^{j \frac{2 \pi}{\lambda} d_{x}(1) \cos \theta_{1}} & \ldots & e^{j \frac{2 \pi}{\lambda} d_{x}(N-1) \cos \theta_{1}} \\
1 e^{j \frac{2 \pi}{\lambda} d_{x}(1) \cos \theta_{2}} & \ldots & e^{j \frac{2 \pi}{\lambda} d_{x}(N-1) \cos \theta_{2}} \\
\vdots & \vdots & \ldots & \vdots \\
1 e^{j \frac{2 \pi}{\lambda} d_{x}(1) \cos \theta_{P}} & \ldots & e^{j \frac{2 \pi}{\lambda} d_{x}(N-1) \cos \theta_{P}}
\end{array}\right]=\mathbf{A D V A}^{H}
$$ 
where

$$
\begin{aligned}
& \mathbf{A}=\left[\mathrm{a}\left(\theta_{1}\right), \mathrm{a}\left(\theta_{2}\right), \ldots, \mathrm{a}\left(\theta_{P}\right)\right] \\
& =\left[\begin{array}{cccc}
e^{-j \frac{2 \pi}{\lambda} d_{x}(1) \cos \theta_{1}} & e^{-j \frac{2 \pi}{\lambda} d_{x}(1) \cos \theta_{2}} & \cdots & 1 \\
\vdots & \vdots & \cdots & e^{-j \frac{2 \pi}{\lambda} d_{x}(1) \cos \theta_{P}} \\
e^{-j \frac{2 \pi}{\lambda} d_{x}(N-1) \cos \theta_{1}} & e^{-j \frac{2 \pi}{\lambda} d_{x}(N-1) \cos \theta_{2}} & \cdots & e^{-j \frac{2 \pi}{\lambda} d_{x}(N-1) \cos \theta_{P}}
\end{array}\right] \\
& \mathbf{D}=\operatorname{diag}\left(d_{1}, d_{2}, \cdots, d_{P}\right) \\
& \mathbf{V}=\operatorname{diag}\left[v\left(\beta_{1}\right), v\left(\beta_{2}\right), \cdots, v\left(\beta_{P}\right)\right] \\
& =\left[\begin{array}{ll}
e^{j \frac{2 \pi}{\lambda} d_{y} \cos \beta_{1}} & \\
e^{j \frac{2 \pi}{\lambda} d_{y} \cos \beta_{2}}
\end{array}\right. \\
& \mathbf{C}_{2}=\mathbf{A D V A}^{H} \\
& =\mathbf{A V D A}^{H} \quad \mathbf{A}^{H} \mathbf{C}_{1} \\
& =\mathbf{A V}\left(\mathbf{A}^{H} \mathbf{A}\right)^{-1}
\end{aligned}
$$$$
e^{\left.j \frac{2 \pi}{\lambda} d_{y} \cos \beta_{P}\right]}
$$

Right multiplying both sides of (17) by $\mathbf{C}_{1}^{\dagger} \mathbf{A}$

$$
\mathbf{C}_{2} \mathbf{C}_{1}^{\dagger} \mathbf{A}=\mathbf{A V}\left(\mathbf{A}^{H} \mathbf{A}\right)^{-1} \mathbf{A}^{H} \mathbf{C}_{1} \mathbf{C}_{1}^{\dagger} \mathbf{A}
$$

Substituted (14) and (15) into (18)

Clearly, Eq. (11) means that the matrix $\mathbf{A}$ is a Vandermonde matrix as long as $\theta_{i},(i=1, \cdots, P)$ comes from different angles. Therefore, $\mathbf{A}$ is a column full-rank matrix, namely, rank $(\mathbf{A})=P$, whose columns are linearly independent. From the expression of $d_{i}$ in (8), we can see that $d_{i}$ is a non-zero constant. Thus, from (12), it is easy to know that the matrix $\mathbf{D}$ is of rank P. Moreover, according to Eq. (13), we confirm that $\mathbf{V}$ satisfies the condition of full rank for different angles $\beta_{i}$.

The eigenvalue decomposition of $\mathbf{C}_{1}$ can be written as

$$
\mathbf{C}_{1}=\sum_{i=1}^{P} \eta_{i} \mathbf{g}_{i} \mathbf{g}_{i}^{H}
$$

where $\left\{\eta_{1}, \ldots, \eta_{P}\right\}$ and $\left\{\mathbf{g}_{1}, \ldots, \mathbf{g}_{P}\right\}$ are the non-zero eigenvalues and corresponding eigenvectors of the matrix $\mathbf{C}_{1}$. The pseudo-inverse of the matrix $\mathbf{C}_{1}$ is

$$
\mathbf{C}_{1}^{\dagger}=\sum_{i=1}^{P} \eta_{i^{-1}} \mathbf{g}_{i} \mathbf{g}_{i}^{H}
$$

Due to the fact that $\mathbf{A}$ is a column full-rank matrix, from (9), we can attain

$$
\mathbf{D} \mathbf{A}^{H}=\left(\mathbf{A}^{H} \mathbf{A}\right)^{-1} \mathbf{A}^{H} \mathbf{C}_{1}
$$

Combining (10) with (16), the alternative expression of $\mathbf{C}_{2}$ can be achieved as follows

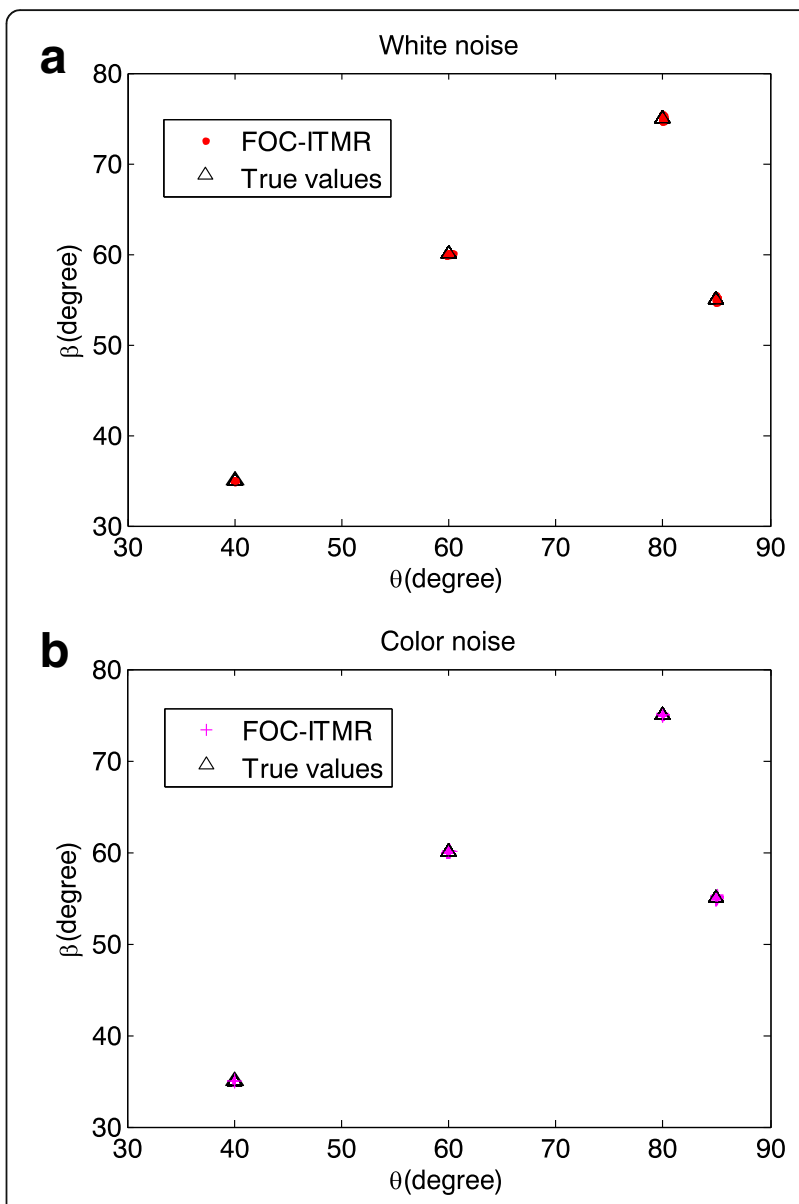

Fig. 2 2-D DOA estimation scattergram. a White noise. b Color noise 


$$
\begin{aligned}
\mathbf{C}_{2} \mathbf{C}_{1}^{\dagger} \mathbf{A} & =\mathbf{A} \mathbf{V}\left(\mathbf{A}^{H} \mathbf{A}\right)^{-1} \mathbf{A}^{H}\left(\sum_{i=1}^{P} \eta_{i} \mathbf{g}_{i} \mathbf{g}_{i}^{H}\right)\left(\sum_{i=1}^{P} \eta_{i^{-1}} \mathbf{g}_{i} \mathbf{g}_{i}^{H}\right) \mathbf{A} \\
& =\mathbf{A} \mathbf{V}\left(\mathbf{A}^{H} \mathbf{A}\right)^{-1} \mathbf{A}^{H} \sum_{i=1}^{P} \mathbf{g}_{i} \mathbf{g}_{i}^{H} \mathbf{A}
\end{aligned}
$$

Notice that $\sum_{i=1}^{P} \mathbf{g}_{i} \mathbf{g}_{i}^{H}$ is an identity matrix, that is $\sum_{i=1}^{P} \mathbf{g}_{i} \mathbf{g}_{i}^{H}=\mathbf{I}$. Thus, Eq. (19) can be further rewritten as

$$
\begin{aligned}
\mathbf{C}_{2} \mathbf{C}_{1}^{\dagger} \mathbf{A} & =\mathbf{A V}\left(\mathbf{A}^{H} \mathbf{A}\right)^{-1}\left(\mathbf{A}^{H} \mathbf{A}\right) \\
& =\mathbf{A} \mathbf{V}
\end{aligned}
$$

From (20), 2-D angle parameters, which are obtained by performing EVD on $\mathbf{C}_{2} \mathbf{C}_{1}^{\dagger}$ denoted as Toeplitz-based generalized DOA matrix, lie in $\mathbf{A}$ and $\mathbf{V}$. By performing EVD on $\mathbf{C}_{2} \mathbf{C}_{1}^{\dagger}$
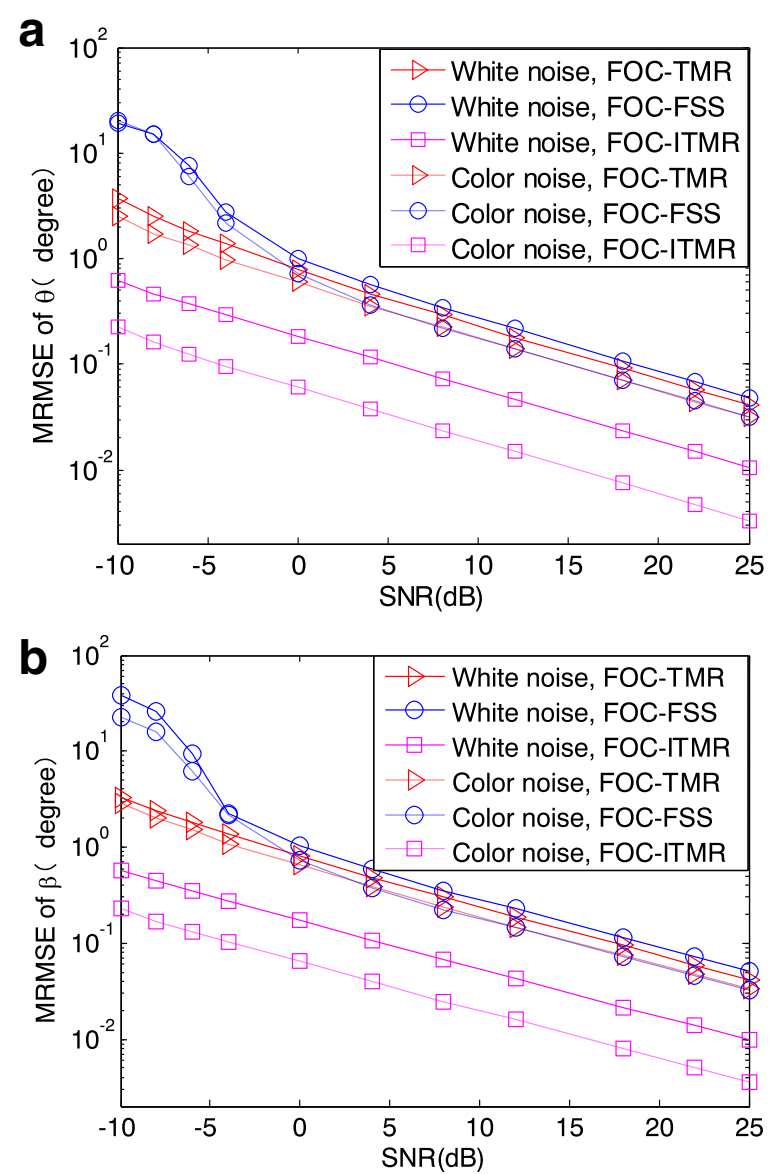

Fig. 3 The MRMSE versus SNR in both white and color noises. a $\theta_{i}$. b $\beta_{i}$

$$
\mathbf{C}_{2} \mathbf{C}_{1}^{\dagger}=\sum_{i=1}^{P} \xi_{i} \mathbf{u}_{i} \mathbf{u}_{i}^{H}
$$

where $\xi_{i}$ and $\mathbf{u}_{i}$ are the non-zero eigenvalues and the corresponding eigenvectors of the matrix $\mathbf{C}_{2} \mathbf{C}_{1}^{\dagger}$. Thus, the $P$ bigger signal subspaces can be obtained from (21). It can be verified that the subspaces spanned by $\mathbf{A}$ and the signal subspaces $\mathbf{U}=\left[\mathbf{u}_{1}, \cdots, \mathbf{u}_{i} \cdots, \mathbf{u}_{P}\right]$ are the same, where $\mathbf{u}_{i}$ is the $\boldsymbol{i}$ th column of the matrix $\mathbf{U}$.

Define $\mathbf{h}_{i}=\mathbf{u}_{i} / \mathbf{u}_{i}(1)$, where $\mathbf{u}_{i}(j),(j=1, \cdots, N)$ denotes the $j$ th element of vector $\mathbf{u}_{i}$, and then we can get

$$
\kappa_{i}=\frac{1}{N-1} \sum_{m=1}^{N-1} \arg \left[\frac{\mathbf{h}_{i}(m+1)}{\mathbf{h}_{i}(m)}\right]
$$

where $\mathbf{h}_{i}(j),(j=1, \cdots, N)$ represents the $j$ th element of vector $\mathbf{h}_{i}$.

Therefore, by combining $\boldsymbol{a}\left(\theta_{i}\right)=$ $\left[1, e^{-j \frac{2 \pi}{\lambda} d_{x} \cos \theta_{i}}, \ldots, e^{-j \frac{2 \pi}{\lambda} d_{x}(N-1) \cos \theta_{i}}\right]^{T}$ and $v\left(\beta_{i}\right)=e^{j \frac{2 \pi}{\lambda} d_{y} \cos \beta_{i}}$, the estimated 2-D DOAs can be obtained
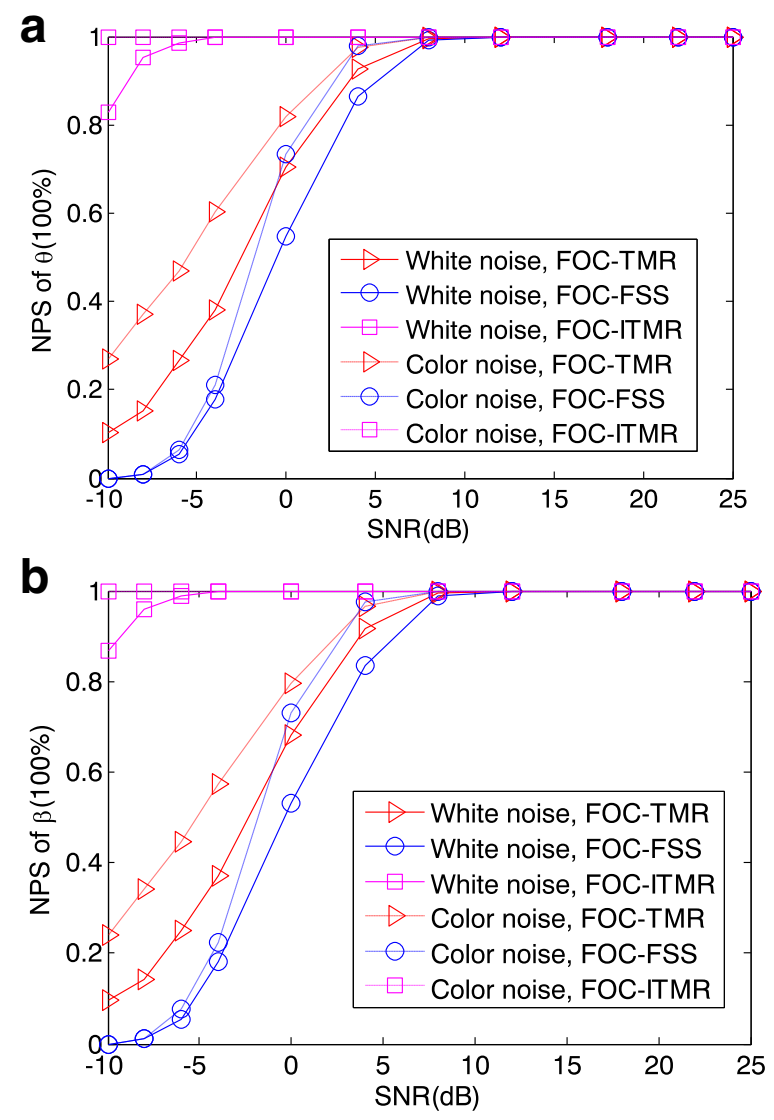

Fig. 4 The NPS versus SNR in both white and color noises. a $\theta_{i}$. b $\beta_{i}$ 


$$
\begin{aligned}
& \theta_{i}=\arccos \left(\frac{\lambda}{2 \pi d_{x}} \kappa_{i}\right) \\
& \beta_{i}=\arccos \left[\frac{\lambda}{2 \pi d_{y}} \arg \left(\xi_{i}\right)\right] .
\end{aligned}
$$

Till now, 2-D DOAs of incoming signals, namely, $\boldsymbol{\theta}_{i}$ and $\boldsymbol{\beta}_{i}$ can be achieved automatically paired according to Eqs. (23) and (24) without additional computations for parameter pair-matching.

The proposed algorithm with finite sampling data can be implemented as follows

Step 1: Compute the cumulant elements $c_{k}^{m}$ and $\tilde{c}_{k}^{m}$ according to (6) and (7), respectively;

Step 2: Reconstruct the two Toeplitz matrices $\mathbf{C}_{1}$ and $\mathbf{C}_{2}$ by (9) and (10);

Step 3: Obtain the pseudo-inverse matrix $\mathbf{C}_{1}^{\dagger}$ by performing the EVD of the matrix $\mathbf{C}_{1}$;
Step 4: Perform EVD of $\mathbf{C}_{2} \mathbf{C}_{1}^{\dagger}$ to obtain the non-zero eigenvalues and the corresponding eigenvector;

Step 5: Estimate the 2-D DOAs of incident coherent source signals via (22)-(24).

\subsection{Location analysis}

In this subsection, the advantage of the proposed algorithm is discussed. As for two $N \times N$ dimension Toeplitz matrices, the maximum number of signals that can be distinguished is $N-1$ by the proposed FOC-ITMR method. Assume that the number of each subarray in [26] is $2 M+1$, the FOC-TMR method can distinguish $M$ signals. According to the parameters set in [25], the FOC-FSS method can tell the same number of signals as [26]. In other words, based on the same array configuration and the same number of sensors, the proposed algorithm has twice larger array aperture than the
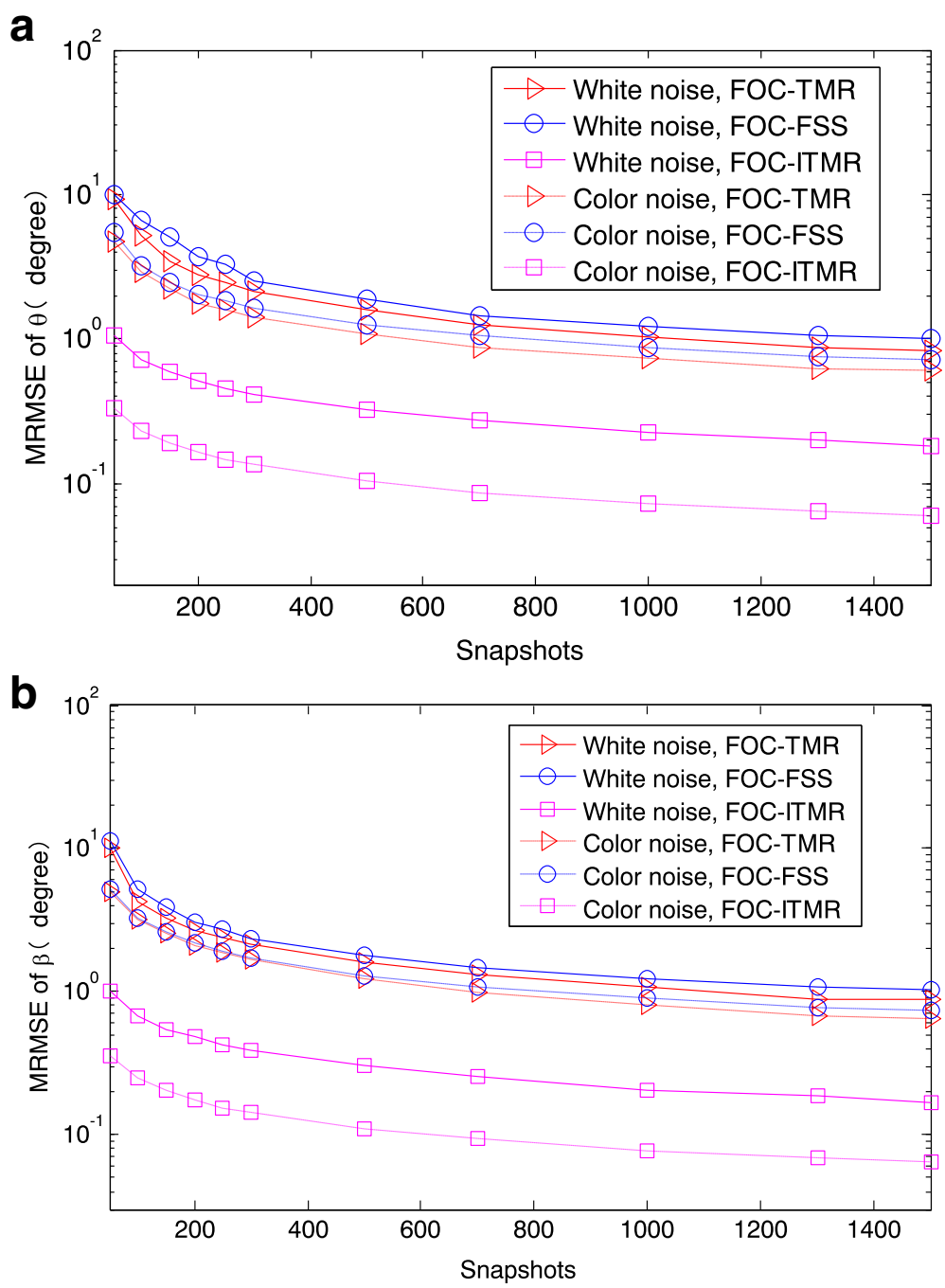

Fig. 5 The MRMSE versus snapshots in both white and color noises. a $\theta_{i}$. b $\beta_{i}$ 
compared FOC-TMR algorithm in [26]. Therefore, the proposed algorithm can not only resolve more signals than the compared method in [26] but also achieve better estimation performance.

\section{Simulation result}

In this section, simulation results are presented to illustrate the validity of the proposed method. We evaluate the performance of the proposed FOC-ITMR algorithm via comparison with the FOC-FSS [25] and the FOC-TMR [26] algorithms with several experiments in both white and color Gaussian noise environments. The color Gaussian noise $z(t)$ is simply considered as bellow [26, 27]

$$
z(\mathrm{t})=n(t)-0.8 n(t-1)+0.7 n(t-2)
$$

where $n(t)$ is white Gaussian noise. Two performance indices, called the maximum root-mean-square error (MRMSE) and normalized probability of success (NPS), are defined to evaluate the performance of the algorithms with respect to variables such as SNR and snapshots.

$$
\text { MRMSE }=\sqrt{\frac{1}{\mathrm{MC}} \sum_{i=1}^{\mathrm{MC}}\left[\max \left(\hat{\sigma}_{i, r}-\sigma_{i}\right)\right]^{2}}
$$

where MC denotes the times of Monte Carlo simulation. $\sigma_{i}$ denotes $\theta_{i}$ or $\beta_{i}$, and $\hat{\sigma}_{i, r}$ is the parameter to be estimated of $\hat{\theta}_{i}$ or $\hat{\beta}_{i}$.

$$
\mathrm{NPS}=\frac{r_{\text {suc }}}{T_{\text {total }}}
$$

where $Y_{\text {suc }}$ and $T_{\text {total }}$ denote the times of success and Monte Carlo trial, respectively. Furthermore, a successful experiment is that satisfies $\max \left(\left|\hat{\theta}_{i}-\theta_{i}\right|\right)<\varepsilon$ or $\max$ $\left(\left|\hat{\beta}_{i}-\beta_{i}\right|\right)<\varepsilon$, where $\varepsilon$ equals 0.8 and 1.8 for comparison versus SNR and snapshots, respectively.

Experiment 1: 2-D DOA estimation performance

Assume that four non-Gaussian signals with $\theta_{i}$ and $\beta_{i}$ impinge from $\left\{40^{\circ}, 80^{\circ}, 60^{\circ}, 85^{\circ}\right\}$ and $\left\{35^{\circ}, 75^{\circ}, 60^{\circ}, 55^{\circ}\right\}$, separately, and the number of sensors in each subarray is $N=5$ with sensor displacement $d_{x}=d_{y}=\lambda / 2$. Figure 2 plots the paired results of four targets from 50 Monte

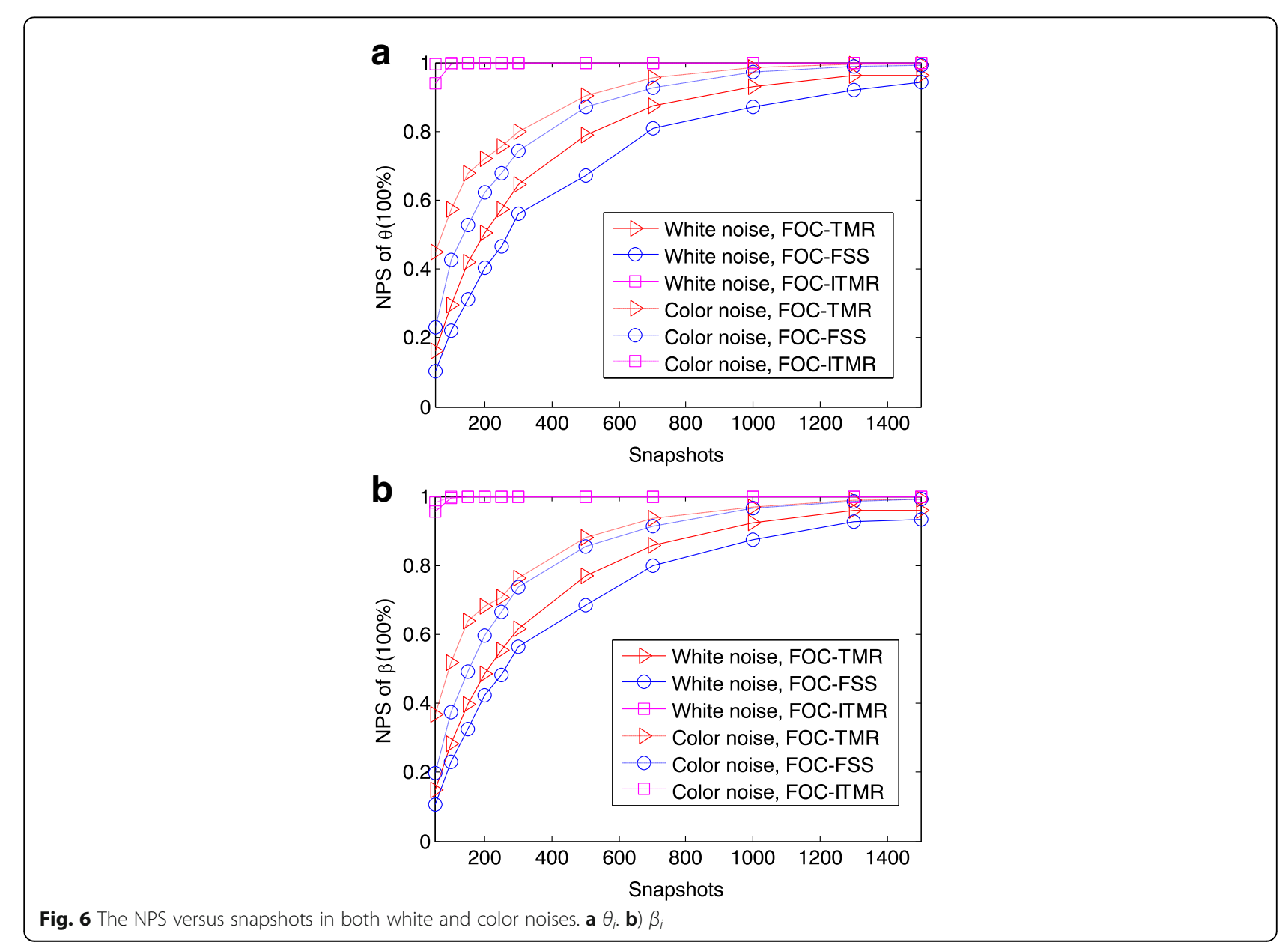




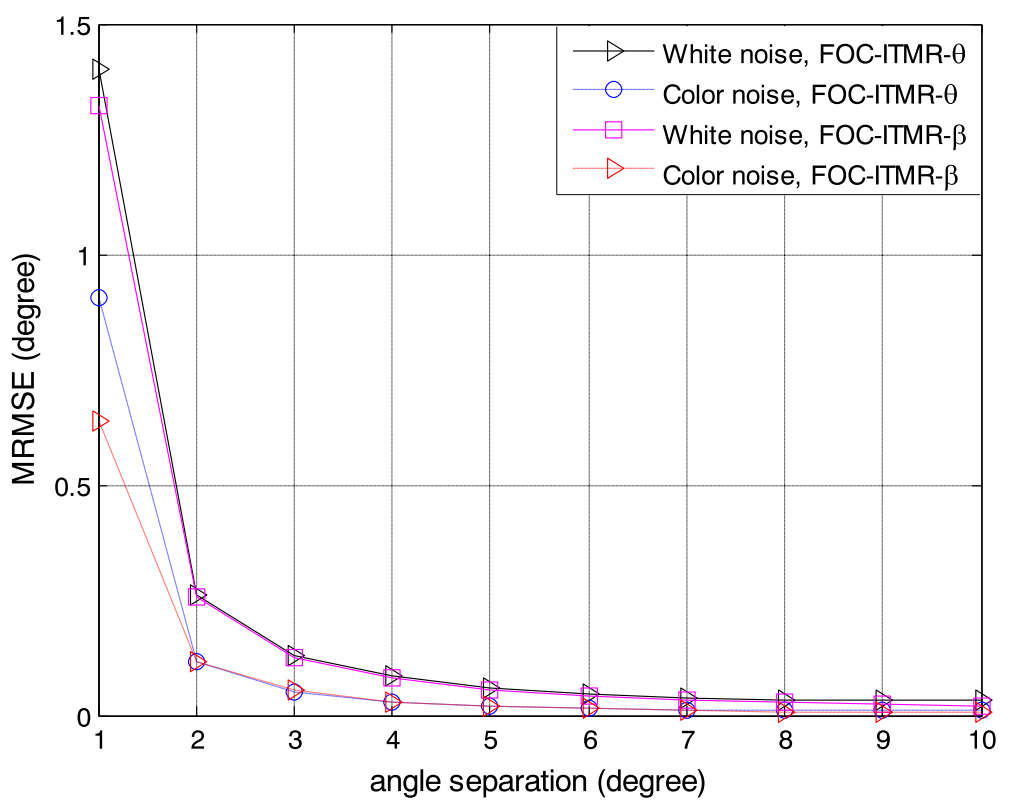

Fig. 7 The MRMSE versus angle separation in both white and color noises

Carlo trials with $\mathrm{SNR}=15 \mathrm{~dB}$ and snapshots $L=1500$, which shows that the 2-D DOA are paired correctly in our scheme in both white and color Gaussian noise situations. On the contrary, the compared FOC-TMR and FOC-FSS methods cannot distinguish them due to array aperture reduced.

Experiment 2: MRMSE and NPS versus SNR

In this simulation, three non-Gaussian signals with $\theta_{i}$ and $\beta_{i}$ are incoming from $\left\{40^{\circ}, 85^{\circ}, 90^{\circ}\right\}$ and $\left\{45^{\circ}, 65^{\circ}, 60^{\circ}\right\}$, separately, and the number of sensors in each subarray is
$N=7$. The number of snapshots is set to $L=1500$, and the SNR is varied from -10 to $25 \mathrm{~dB}$. The $\theta_{i}$ and $\beta_{i}$ MRMSE curves of the proposed method and the FOC-TMR and FOC-FSS methods versus SNR are shown in Fig. 3, where 2000 Monte Carlo trials are used. Figure 3 illustrates that the proposed method has much lower MRMSE than the compared FOC-TMR and FOC-FSS algorithms in both spatially white noise and spatially color noise environments, especially at low SNR. Figure 4 illustrates the NPS of the DOAs versus SNR, which illustrates that the

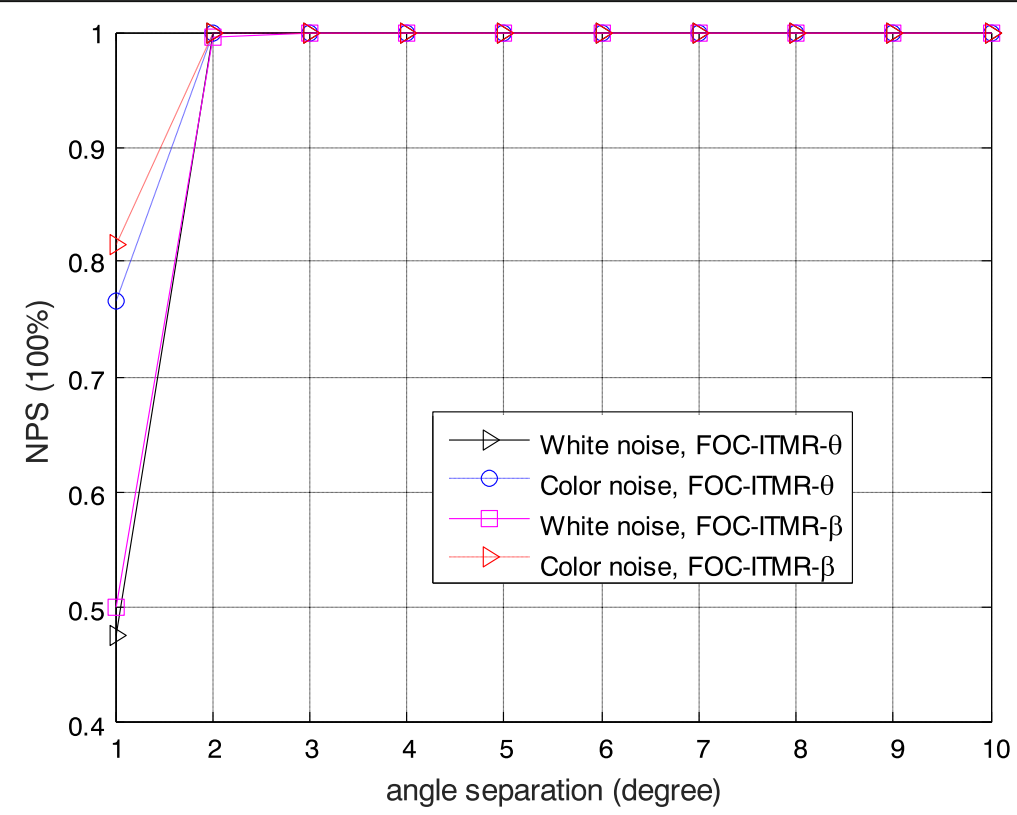

Fig. 8 The NPS versus angle separation in both white and color noises 
performance of the proposed method is better than that of FOC-TMR and FOC-FSS algorithms as the SNR increases. The reason is that the proposed algorithm utilizes the whole ULAs to estimate the source signals, which avoids the loss of array physical aperture and achieves excellent decoherence performance.

Experiment 3: MRMSE and NPS versus snapshots

The third simulation considers the same scenario as the second one at different number of snapshots. When the SNR is $0 \mathrm{~dB}$, the MRMSE of the estimated parameters against number of snapshots is shown in Fig. 5. It can be seen from Fig. 5 that the proposed method achieves higher estimation accuracy than the FOC-TMR and FOC-FSS algorithms as the number of snapshots increases.

In addition, the NPS of the DOAs signals against the number of snapshots is shown in Fig. 6 when the SNR is $0 \mathrm{~dB}$. From Fig. 6, we can see that the performance of the proposed algorithm is superior to the compared methods as the number of snapshots is varied from 0 to 500. That is, in small number of snapshots, the superiority of the proposed algorithm is more pronounced. This indicates that the proposed algorithm will be more useful when the low-computational cost and highly realtime data process are required.

Experiment 4: MRMSE and NPS versus angle separation

The fourth simulation studies the estimation performance of the proposed algorithm between $\theta$ and $\beta$. The number of snapshots is 1500 , and the input SNR is $10 \mathrm{~dB}$. Consider three non-Gaussian signals with 2-D DOAs $\theta$ and $\beta$ coming from $\theta=\left[40^{\circ}, 85^{\circ}, 85^{\circ}+\right.$ detra $]$ and $\beta=\left[45^{\circ}, 60^{\circ}+\right.$ detra, $\left.60^{\circ}\right]$, where the "detra" denotes the angle separation, varying from $1^{\circ}$ to $10^{\circ}$. The simulation experiments are based on 200 Monte Carlo trials.

The MRMSE of the 2-D DOAs versus angle separation has been shown in Fig. 7. It can be observed from Fig. 7 that no matter in white Gaussian noise situation or in color Gaussian noise situation, the MRMSE of $\theta$ achieves a similar performance to the MRMSE of $\beta$. Figure 8 shows the NPS of the 2-D DOAs versus angle separation. Apparently, the same conclusions can be drawn. Figure 8 illustrates that the performance of $\theta$ is approximately identical to that of the $\beta$ in both white and color Gaussian noise situations.

\section{Conclusions}

In this paper, a novel FOC-ITMR method for coherent signals estimation is proposed. The whole information of the two parallel ULAs is fully utilized by changing the reference element to reconstruct the two new cumulantsbased matrices, which avoids the loss of array physical aperture. Therefore, the proposed method gains excellent decoherence performance. Simulation results show the validity of the presented FOC-ITMR algorithm.

\section{Acknowledgements}

This research was supported by the National Natural Science Foundation of China (61602346), by the Key Talents Project for Tianjin University of Technology and Education (TUTE) (KYQD16001), by the Tianjin municipal science and technology innovation platform, intelligent transportation coordination control technology service platform (16PTGCCX00150) and by the National Natural Science Foundation of China (61601494).

\section{Competing interests}

The authors declare that they have no competing interests.

\section{Publisher's Note}

Springer Nature remains neutral with regard to jurisdictional claims in published maps and institutional affiliations.

\section{Author details}

${ }^{1}$ School of Automotion and Transportation, Tianjin University of Technology and Education, Tianjin 300222, China. ${ }^{2}$ Tianjin Key Laboratory of Wireless Mobile Communications and Power Transmission, Tianjin Normal University, Tianjin 300387, China. ${ }^{3}$ School of Electronic Engineering, Tianjin University of Technology and Education, Tianjin 300222, China. ${ }^{4}$ School of Electronic Information Engineering, Tianjin University, Tianjin 300072, China. ${ }^{5}$ College of Electronic and Communication Engineering, Tianjin Normal University, Tianjin 300387, China.

Received: 14 November 2016 Accepted: 28 February 2017 Published online: 20 March 2017

\section{References}

1. H Krim, M Viberg, Two decades of array signal processing research: the parametric approach. IEEE Signal Process Mag 13(4), 67-94 (1996)

2. Z Li, K Liu, Y Zhao et al., MaPIT: an enhanced pending interest table for NDN with mapping bloom filter. IEEE Commun Lett 18(11), 1423-1426 (2014)

3. Z Li, L Song, H Shi, Approaching the capacity of K-user MIMO interference channel with interference counteraction scheme. Ad Hoc Netw 2016, 1-6 (2016)

4. Z Z Li, Y Chen, $H$ Shi et al., NDN-GSM-R: a novel high-speed railway communication system via named data networking. EURASIP J Wirel Commun Netw 2016(48), 1-5 (2016)

5. X Liu, Z Li, P Yang et al., Information-centric mobile ad hoc networks and content routing: a survey. Ad Hoc Netw 2016, 1-14 (2016)

6. H Chen, C Hou, Q Wang et al., Improved azimuth/elevation angle estimation algorithm for three-parallel uniform linear arrays. IEEE Antennas Wirel Propag Lett 14, 329-332 (2015)

7. $\mathrm{H}$ Wu, C Hou, $\mathrm{H}$ Chen et al., Direction finding and mutual coupling estimation for uniform rectangular arrays. Signal Process 117, 61-68 (2015)

8. J Liang, D Liu, Joint elevation and azimuth direction finding using L-shaped array. IEEE Trans Antennas Propag 58(6), 2136-2141 (2010)

9. S Kikuchi, H Tsuji, A Sano, Pair-matching method for estimating 2-D angle of arrival with a cross-correlation matrix. IEEE Antennas Wirel Propag Lett 5(1), 35-40 (2006)

10. N Tayem, HM Kwon, Azimuth and elevation angle estimation with no failure and no eigen decomposition. Signal Process 86(1), 8-16 (2006)

11. RO Schmidt, Multiple emitter location and signal parameter estimation. IEEE Trans Antennas Propag 34(3), 276-280 (1986)

12. R Roy, T Kailath, ESPRIT-estimation of signal parameters via rotational invariance techniques. IEEE Trans Acoust Speech Signal Process 37(7), 984-995 (1989)

13. Z Shan, TP Yum, A conjugate augmented approach to direction-of-arrival estimation. IEEE Trans Signal Process 53(11), 4104-4109 (2005)

14. WJ Zeng, XL Li, XD Zhang, Direction-of-arrival estimation based on the joint diagonalization structure of multiple fourth-order cumulant matrices. IEEE Signal Process Lett 16(3), 164-167 (2009)

15. TH Liu, JM Mendel, Azimuth and elevation direction finding using arbitrary array geometries. IEEE Trans Signal Process 46(7), 2061-2065 (1998)

16. J Liang, Joint azimuth and elevation direction finding using cumulant. IEEE Sensors J 9(4), 390-398 (2009)

17. $\mathrm{P}$ Chevalier, L Albera, A Ferreol et al., On the virtual array concept for higher order array processing. IEEE Trans Signal Process 53(4), 1254-1271 (2005)

18. E Gonen, JM Mendel, MC Dogan, Applications of cumulants to array processing-part iv: direction finding in coherent signals case. IEEE Trans Signal Process 45(9), 2265-2276 (1997) 
19. N Tayem, HM Kwon, L-shape 2-dimensional arrival angle estimation with propagator method. IEEE Trans Antennas Propag 53(5), 1622-1630 (2005)

20. SU Pillai, BH Kwon, Forward/backward spatial smoothing techniques for coherent signal identification. IEEE Trans Acoust Speech Signal Process 37(1), 8-15 (1989)

21. F Chen, S Kwong, CW Kok, ESPRIT-like two-dimensional DOA estimation for coherent signals. IEEE Trans Aerosp Electron Syst 46(3), 1477-1484 (2010)

22. GM Wang, JM Xin, NN Zheng et al., Computationally efficient subspacebased method for two-dimensional direction estimation with L-shaped array. IEEE Trans Signal Process 59(7), 3197-3212 (2011)

23. X Nie, LP Li, A computationally efficient subspace algorithm for 2-D DOA estimation with L-shaped array. IEEE Signal Process Lett 21(8), 971-974 (2014)

24. JF Gu, WP Zhu, MNS Swamy, Joint 2-D DOA estimation via sparse L-shaped array. IEEE Trans Signal Process 31(5), 1171-1182 (2015)

25. G Du, N Tong, Y Wang et al., Joint estimation of frequency and 2-D arrival angles of coherent signals in the presence of colored noise. Congr Image Signal Process 5, 281-285 (2008)

26. H Chen, C Hou, Q Wang et al., Cumulants-based Toeplitz matrices reconstruction method for 2-D coherent DOA estimation. IEEE Sensors J 14(8), 2824-2832 (2014)

27. JF Chen, XL Zhu, XD Zhang, A new algorithm for joint range DOA-frequency estimation of near-field sources. EURASIP J Appl Signal Process 2004(3), 386-392 (2004)

\section{Submit your manuscript to a SpringerOpen ${ }^{\circ}$ journal and benefit from:}

- Convenient online submission

- Rigorous peer review

- Immediate publication on acceptance

Open access: articles freely available online

- High visibility within the field

- Retaining the copyright to your article

Submit your next manuscript at $>$ springeropen.com 